houses, the occupants of the cellars and basements are not only exposed to the dangers of gas poisoning against which the gas mask offers no protection, but they run the risk of gas explosions and fires. During the war in air raids on cities and towns and villages serious gas explosions and fires occurred and many lost their lives.

The gas thus produced may be ignited by the great heat resulting from the detonation of the bomb, or by incendiary bombs, or by the presence of a naked light. As bombs penetrate deeply before detonating gas may be given off from the ground slowly, and very gradually collect in cellars and basements, to be fired by someone approaching with a naked light; sometimes it may be hours after the bombardment has ceased. In other cases gas has found its way into cellars at a distance from the exploding bomb by passing through fissures that have been caused by the explosion. Serious gas explosions and fires were also caused by the ignition of illuminant gas that had escaped from pipes smashed by the explosion. These explosions may occur during salvage work among the debris, when pockets of gas may be fired.

It is an obvious precaution to make use of electric torches during all operations where gas is suspected, and, after an air raid, never to enter a cellar with a naked light.

\section{THE STRETCHER PROBLEM}

BY

\section{CROME, B.Com., L.R.C.P. \& S.Ed.}

\section{Formerly Chief Medical Officer, XVth Spanish Army Corps}

No single item in the equipment of the medical services in time of war is more important than the stretcher. In the Spanish War, as a result of extensive experience, one type was universally adopted for use in the armed services and in the civilian casualty organizations. This stretcher was employed in the field for the primary transport of casualties from the line of fire to the dressing stations. The same stretcher was carried in all ambulances, and was used in the waiting rooms of field and base hospitals and in all medical units connected with A.R.P.

At present there are two main types of stretcher in existence in England. One is the standard R.A.M.C. stretcher and the other is the type recommended by the Ministry of Health for A.R.P. purposes. The fact that the Ministry of Health has recommended a stretcher entirely different from that used by the R.A.M.C. points to the conclusion that the latter was not considered satisfactory; while the fact that the R.A.M.C. has not adopted, and is unlikely to adopt, the stretcher recommended by the Ministry of Health must imply that neither of the two types is entirely adequate. Before describing the stretcher used in Spain it is necessary to point out that stretchers serve three main and two subsidiary purposes. They are used (1) to carry wounded from the field to the ambulance base; (2) in motor vehicles and trains for the transport of wounded to and from hospitals; and (3) in the waiting rooms of dressing stations and hospitals for the interim accommodation and treatment of patients before operation or hospitalization. The secondary uses of stretchers are (a) as sleeping accommodation for medical personnel; and (b) for the erection of huts, shelters, covers, and camouflage in circumstances where no other building material is at hand.

\section{General Specification}

Stretchers must have definite qualities, which will now be stated in their approximate order of importance.

1. Stretchers. must be as light as is compatible with serviceability.
2. Stretchers must be comfortable. At times patients will have to lie on them for twenty-four hours or more.

3. Stretchers must be robust; they will have to stand up to the hardest wear.

4. After gas attacks stretchers may have to be decontaminated. It is essential to use material which will stand decontamination.

5. Although not much can be done in the way of postural treatment during transport, it is helpful to have a stretcher capable of holding some part of the patient's body in a position of elevation. This is especially important in cases of cranial or thoracic injury.

6. Stretchers must have sufficient clearance to prevent the patient from touching the ground. If a patient is at a sufficient distance from the ground, treatment, such as injections, bandaging, blood transfusion, is considerably easier.

7. Stretchers must be used in motor vehicles, not all of which have been constructed as ambulances. The minimum of structural alteration of the vehicle itself should be necessary before it is adapted to carry stretchers.

8. Stretchers must have suitable attachments for the application of essential splints, such as the Thomas splint.

9. While not in use for the transport of casualties the stretchers will have to be carried by stretcher-bearers from one place to another. It is necessary to make this work as easy as possible. At times only one man will be available to carry such a stretcher.

\section{Need for Standardization}

Two essential considerations in the transport of casualties are the prevention of shock and the prevention of further local injury at the site of the lesion. All extra handling of the patient, such as is necessary for the changing of stretchers, increases the danger of shock and of further injury. The ideal to aim at is, therefore, complete and uninterrupted transport of the casualty on one stretcher. At each break in the evacuation journey the loaded stretciner should be exchanged for an empty one, the latter being returned to the previous point by the returning stretcher-bearers or ambulance. Thus the patient will be spared the pain, discomfort, and danger of being moved from one stretcher to another, and much time will be saved in the process of evacuation. Ambulances will be able to return immediately after depositing their casualties and receiving in exchange an equal number of empty stretchers with the corresponding number of blankets. Such a system of relay evacuation is only possible if the same type of stretcher is used throughout the evacuation line. The needs of the armed services and of the civilian population are identical in respect of stretchers, and there is no reason why the same type of stretcher should not be used throughout the country.

If a "universal "type of stretcher is necessary from the functional point of view its standardization is essential from the point of view of production, maintenance, and replacement. The stretcher requirements of the armed forces may be calculated from the presumed ratio of one stretcher to thirty men. For the civilian population the ratio is more variable, but an anticipated figure of 500,000 stretchers at the beginning of the war, going up to 750,000 during the first year, is not an exaggeration. The problem of production on this scale and continuous replacement can only be satisfactorily solved by complete standardization and the timely preparation of raw materials necessary in the construction of these stretchers. It is also desirable that if one of the component parts of a stretcher is irreparably damaged or lost it should be capable of being replaced by another identical part without the necessity for discarding the entire stretcher.

Storage space must also be considered. Stretchers must be produced in peace time and stored, and they must be delivered in such a way that the minimum space is 
required for their transport and storage. Finally, cost can only be brought down to a minimum if the stretchers are standardized on a mass production basis.

\section{The Spanish Stretcher}

The Spanish stretcher consists of five parts which can be readily assembled: (1) two horizontal wooden poles ; (2) iron head-piece; (3) iron foot-piece ; (4) a double layer of canvas fastened with leather straps in front of the head-piece ; and (5) two shoulder-slings made of stout leather with loops for the handles of the stretcher. When assembled, the stretchel is carried by two bearers, each wearing a shoulder-sling in such a way that the weight of the stretcher is secured by these slings even if the hand grip should fail (Fig. 1). Dismantled head- and footpieces can be rolled into the canvas and carried on the shoulders of one of the stretcher-bearers, being secured by the shoulder-slings. If necessary, the same stretcherbearer can also carry the poles (Fig. 2), but it is usual for the poles to be left to the second stretcher-bearer.

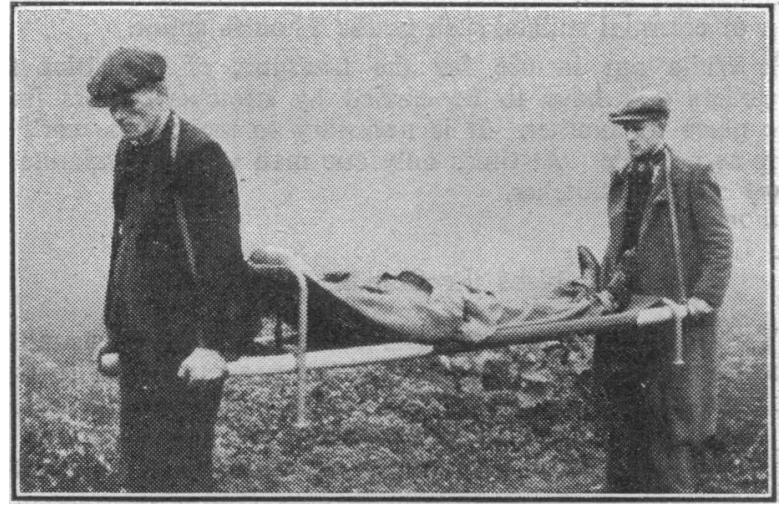

Fig 1.-The Spanish stretcher in use.

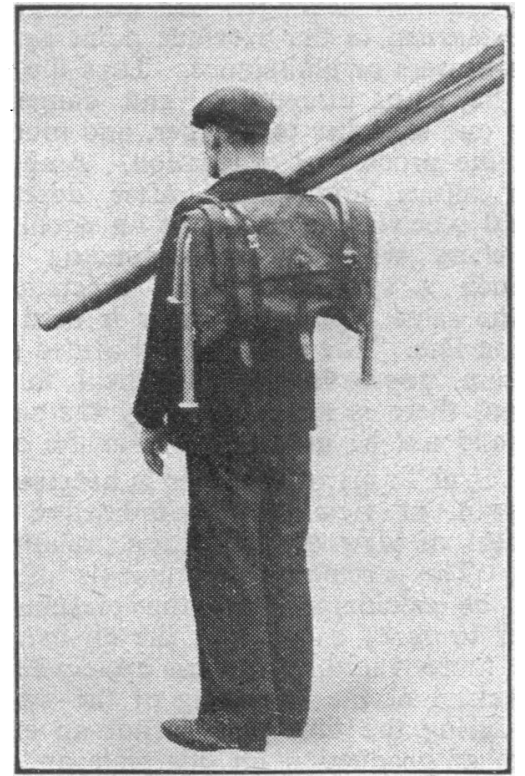

FIG. 2.-The Spanish stretcher dismantled.

The fittings necessary for the reception of the stretcher in a motor vehicle can also be used for stretchers which are not of the type described (Fig. 3). These fittings are inexpensive and easy to manufacture, and can be fitted in any closed van without structural alteration. Each fitting has two swivels which enable it to be swung flat against the side of the ambulance, leaving space free for sitting patients if this is required. Though it serves as a spring, the fitting is extremely strong. During the whole of the Spanish War it was unknown for any of them to

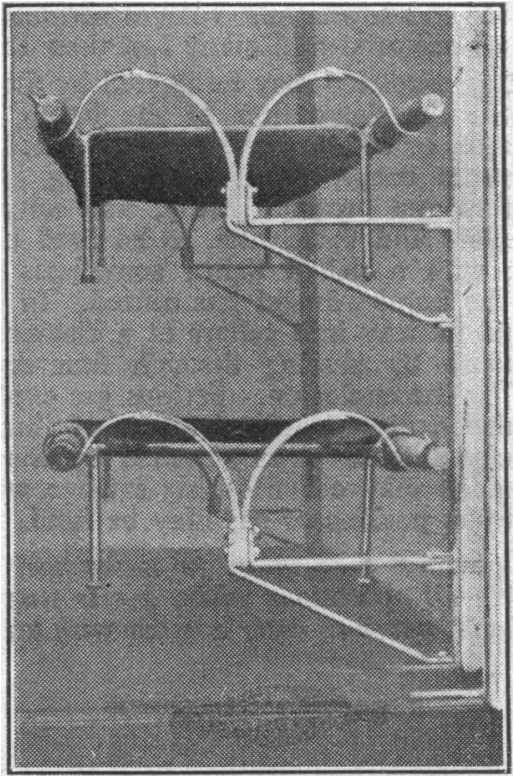

FIG. 3. - The fittings used to convert an ordinary light van for use as an ambulance.

give way. Moreover, it has a very small surface and is therefore seldom damaged by fragments of bombs or shells.

\section{Conclusion}

It is suggested that the type of stretcher described can be put to all uses in war time. It meets all the requirements for such stretchers and is easily standardized.

By reversing the position of the patient on the stretcher the upper horizontal bar of the head-piece may be used for attachment of a Thomas's splint. The empty triangular space formed by the upper and lower layers of the canvas at the attachment to the head-piece may be used for carrying the patients' loose belongings.

All the component parts of the stretcher, including the canvas, are detachable and can easily be cleaned, washed, disinfected, and decontaminated.

The incline formed by the upper layer of the canvas allows for postural treatment. The complete stretcher weighs $25 \mathrm{lb}$.

While I have not attempted to balance the advantages and disadvantages of the suggested stretcher against those used by the R.A.M.C. or recommended by the Ministry of Health, its relative merits will be readily appreciated by all who are familiar with the problem.

I wish to express my gratitude to Messrs. N. A. Bronsten and Co. Ltd. for their good offices in preparing the demonstration model of the stretcher described above and allowing me to reproduce the photographs.

Photodynamic Activity in Carcinogenic Hydrocarbons.Exposure to extreme dilutions of either of four carcinogenic hydrocarbons sensitizes cultures of Paramoecium to ultraviolet light. Other substances having this photodynamic action (eosin, acriflavine, etc.) are less potent and unaffected by light conditions during contact, whereas carcinogenic hydrocarbons sensitize much more effectively in the dark. These observations, apart from their wider theoretical interest, disclose a method for the biological assay of small quantities of carcinogenic hydrocarbons.-Comparison of Photodynamic Activity of Some Carcinogenic with Non-Carcinogenic Compounds. I. Doniach.-Brit. J. exp. Path. June 1939, 20, 227. 